\title{
Improving IMRT delivery efficiency using intensity limits during inverse planning
}

Martha M. Coselmon, ${ }^{\text {a) }}$ Jean M. Moran, Jeffrey D. Radawski, and Benedick A. Fraass

Department of Radiation Oncology, University of Michigan, Ann Arbor, Michigan 48109

(Received 15 November 2004; revised 18 February 2005; accepted for publication 18 February 2005; published 12 April 2005)

Inverse planned intensity modulated radiotherapy (IMRT) fields can be highly modulated due to the large number of degrees of freedom involved in the inverse planning process. Additional modulation typically results in a more optimal plan, although the clinical rewards may be small or offset by additional delivery complexity and/or increased dose from transmission and leakage. Increasing modulation decreases delivery efficiency, and may lead to plans that are more sensitive to geometrical uncertainties. The purpose of this work is to assess the use of maximum intensity limits in inverse IMRT planning as a simple way to increase delivery efficiency without significantly affecting plan quality. Nine clinical cases (three each for brain, prostate, and head/neck) were used to evaluate advantages and disadvantages of limiting maximum intensity to increase delivery efficiency. IMRT plans were generated using in-house protocol-based constraints and objectives for the brain and head/neck, and RTOG 9406 dose volume objectives in the prostate. Each case was optimized at a series of maximum intensity ratios (the product of the maximum intensity and the number of beams divided by the prescribed dose to the target volume), and evaluated in terms of clinical metrics, dose-volume histograms, monitor units (MU) required per fraction (SMLC and DMLC delivery), and intensity map variation (a measure of the beam modulation). In each site tested, it was possible to reduce total monitor units by constraining the maximum allowed intensity without compromising the clinical acceptability of the plan. Monitor unit reductions up to $38 \%$ were observed for SMLC delivery, while reductions up to $29 \%$ were achieved for DMLC delivery. In general, complicated geometries saw a smaller reduction in monitor units for both delivery types, although DMLC delivery required significantly more monitor units in all cases. Constraining the maximum intensity in an inverse IMRT plan is a simple way to improve delivery efficiency without compromising plan objectives. (C) 2005 American Association of Physicists in Medicine.

[DOI: $10.1118 / 1.1895545]$

\section{INTRODUCTION}

The introduction of inverse planned intensity modulated radiotherapy (IMRT) into clinics has allowed improvement in many areas of therapy, including dose escalation, reduction of normal tissue toxicity, simultaneous boost treatments, and a high degree of dose conformity and coverage that may not have been possible with conventional three-dimensional (3D) conformal therapy. ${ }^{1-9}$ Such improvements are possible not just because of the ability of inverse planning optimization systems to create steep dose gradients between target and normal tissue interfaces, but also because of their ability to place dose in a nonintuitive fashion (compared to a forward planned technique). Since inverse planning is a discrete optimization problem with many variables, IMRT beams have the potential to be discontinuous, with very sharp gradients over small distances. On one hand, these features are considered desirable because they help achieve the objectives given to the treatment planning system. On the other hand, these features can lead to undesirable effects, including large increases in monitor units (MU), sensitivity to geometric uncertainties, inaccurate leaf sequencing, and prolonged delivery times. ${ }^{10-13}$ Another concern is the additional dose delivered to the patient from transmission and leakage as MU are increased. ${ }^{14,15}$ As intensity patterns become more complex, the differences between the computed, sequenced, and delivered intensity patterns may increase. This may become an important issue as those involved in the planning process gain more experience and use increasingly strict specifications in the objective function.

The difficulties associated with complex intensity patterns have motivated attempts to increase the efficiency of IMRT delivery as well as to reduce unnecessary modulation in delivered IMRT beams. ${ }^{10}$ To prevent cases of inaccurate sequencing and make the optimization process more efficient with respect to delivery, IMRT delivery constraints have been incorporated directly into the optimization process $^{12,16-18}$ and improvements in efficiency have been obtained with advances in leaf sequencing algorithms. ${ }^{19-23}$ Much effort has also been devoted to incorporation of smoothing algorithms during or after optimization, to produce more continuous intensity patterns that reduce undeliverability as well as excessive MU..$^{10,11,13,24-26}$

Another possible strategy is to acknowledge that some of the high intensity peaks may result from limitations in the inverse planning optimization strategy and may not be essential for high quality plans. If so, then constraining the maximum allowable intensity for an IMRT plan may inhibit the optimization engine from pursuing an undesirable path that may be an artifact of the point-based inverse planning ap- 
proach. Using a maximum intensity limit for the plan in the optimization process can result in beamlet patterns that lack potentially unnecessary modulation and sharp spikes, while still allowing the optimization algorithm to make the proper tradeoffs between target and normal tissue doses. This approach, which is the focus of the current work, would ideally produce a plan that will be sequenced more accurately, delivered with fewer MUs, and less sensitive to positioning errors than an IMRT plan derived without maximum intensity limits, while still achieving the defined clinical objectives. The purpose of this paper is to evaluate both the delivery improvements and changes in plan quality that result from applying intensity limits during inverse plan optimization to a series of prostate, brain, and head/neck plans.

\section{METHODS}

All inverse planning and analysis for this study was performed with our in-house-developed 3D planning (UMPLAN) $^{27-31}$ and inverse planning (UMOPT) ${ }^{32,33}$ systems. All cases were optimized with $6 \mathrm{MV}$ beamlet IMRT planned for a $6 \mathrm{MV}$ linear accelerator (Varian Medical Systems, $21 \mathrm{EX}$ ) with 120 leaf multileaf collimator (MLC) (0.5 and 1.0 $\mathrm{cm}$ leaf widths). Dose calculations for the inverse planning system were performed with a convolution/superposition algorithm derived from the work of Mackie. ${ }^{34}$ All cases were optimized using a quasi-Newton-based search strategy, incorporating dose, dose-volume, and biological-based costlets designed to meet each of the specified planning protocols. Simulated annealing was also used, to ensure that the optimized plans did not represent local minima of the cost function. Leaf sequencing for SMLC delivery was performed with an in-house-developed leaf sequencer based on the method reported by Bortfeld et al., ${ }^{35}$ using the parameters routinely selected for our clinical IMRT plans. Delivery sequences can be up to 250 segments per beam, with the goal of achieving a correspondence between planned and delivered intensities of $1.0 \%$. Similarly, plans were sequenced for dynamic delivery using an in-house sequencer with partial leaf synchronization and a 150 segment limit. $22,36-40$

To assess the value of limiting maximum intensity in an effort to improve delivery efficiency in IMRT, we tested the procedure in three different clinical sites: brain, prostate, and head/neck. The sites and specific plans were chosen to represent a range of complexity and clinical tradeoffs between target coverage and normal tissue sparing. Three cases each were studied for each site (nine cases total). After applying clinical-based cost functions, each plan was optimized using a series of assigned values of the maximum beamlet intensity. Each plan was also optimized without using a maximum intensity limit to represent the unconstrained solution. In the text, these plans are referred to as the unconstrained intensity plans and the maximum intensity ratio [see Eq. (1)] is calculated based upon the highest occurring beamlet value in the plan. Because the required maximum intensity per beamlet is technique and dose prescription dependent, the maximum intensity ratio was chosen as a metric to permit direct compari- son of plans with different prescriptions or number of beams. The "maximum intensity ratio" or MIR, is defined as

$$
\operatorname{MIR}=\frac{I_{\max } N_{b}}{D_{t}},
$$

where $I_{\max }$ is the maximum intensity allowed for each beamlet, $N_{b}$ is the number of beams in the plan, and $D_{t}$ is the prescribed dose to the target volume. In this study, $I_{\max }$ is set for a plan so that no beamlet defined in the plan can exceed the maximum intensity. Note that the utility of the maximum intensity ratio for comparing plans from treatment sites with large geometrical differences will be limited.

Another metric, the intensity map variation, was defined for each field and used to measure the modulation across a beam. The plan intensity map variation (PIMV) is calculated by summing the variation for each field and is defined for each plan as

$$
\begin{aligned}
\mathrm{PIMV}= & \sum_{n=1}^{N_{b}}\left[\sum _ { j = 1 } ^ { J - 1 } \sum _ { k = 1 } ^ { K - 1 } \left(\left|I_{j k}-I_{j, k+1}\right|\right.\right. \\
& \left.\left.+\left|I_{j k}-I_{j+1, k}\right|+\left|I_{j k}-I_{j+1, k+1}\right|\right)\right],
\end{aligned}
$$

where $N_{b}$ is again the number of beams in the plan, $J$ is the maximum number of beamlets in the direction parallel to the motion of the MLC, $K$ is the maximum number of beamlets in the direction perpendicular to the motion of the MLC, and $I_{j k}$ is the intensity of the beamlet at the $(j, k)$ grid position. We have chosen the PIMV to be a measurement of the field modulation that is not biased by the sequencing algorithm chosen. For this study, each beam was defined as a regular grid, however, if a beam was defined as segments or an irregular grid, it would be necessary to apply a grid based on the smallest beamlet dimension and then use Eq. (2) to calculate the PIMV.

For a measure of the similarity between two intensity maps for plans at different maximum intensity ratios, we computed the correlation coefficient for each intensity grid with respect to the unconstrained optimized intensity grid. Thus, for one beam, given an optimal intensity map at a maximum intensity ratio of $A$, and another optimal intensity map at a maximum intensity ratio of $B$, we can define the correlation coefficient as

$$
C_{I_{A} I_{B}}=\frac{\sum_{j} \sum_{k}\left(I_{A, j k}-\overline{I_{A}}\right)\left(I_{B, j k}-\overline{I_{B}}\right)}{\sqrt{\left(\sum_{j} \Sigma_{k}\left(I_{A, j k}-\overline{I_{A}}\right)^{2}\right)\left(\sum_{j} \Sigma_{k}\left(I_{B, j k}-\overline{I_{B}}\right)^{2}\right)}},
$$

where $j$ and $k$ are the dimensions of the intensity map [as discussed in Eq. (2)], $I_{X j k}$ is the intensity of grid element $(j, k)$ when the maximum intensity ratio is $X$, and $\overline{I_{X}}$ is the mean intensity of grid $I_{X}$. The correlation coefficient may vary from -1.0 to 1.0. A value of 1.0 means that the two patterns are perfectly linearly and positively correlated, while a value of -1.0 means that the two patterns are perfectly linearly and oppositely correlated. A high absolute number means there is a high level of correlation, while a small absolute number represents a weak correlation. 
TABLE I. Beam angle information for each case.

\begin{tabular}{|c|c|c|c|}
\hline Case(s) & $\operatorname{Gantry}\left({ }^{\circ}\right)$ & Collimator $\left({ }^{\circ}\right)$ & Table $\left(^{\circ}\right)$ \\
\hline Brain 1 & {$\left[\begin{array}{llllll}270 & 310 & 300 & 240 & 45\end{array}\right]$} & {$\left[\begin{array}{lllll}0 & 0 & 0 & 0 & 0\end{array}\right]$} & {$\left[\begin{array}{lllll}0 & 315 & 270 & 270 & 55\end{array}\right]$} \\
\hline Brain 2 & {$\left[\begin{array}{lllll}270 & 270 & 270 & 90\end{array}\right]$} & {$\left[\begin{array}{lllll}280 & 270 & 270 & 90\end{array}\right]$} & {$\left[\begin{array}{lllll}0 & 335 & 290 & 65\end{array}\right]$} \\
\hline Brain 3 & {$\left[\begin{array}{lllll}215 & 285 & 120 & 70\end{array}\right]$} & {$\left[\begin{array}{llll}0 & 0 & 0 & 0\end{array}\right]$} & {$\left[\begin{array}{llll}0 & 0 & 70 & 70\end{array}\right]$} \\
\hline Prostate $1,2,3$ & {$\left[\begin{array}{lllllllll}0 & 40 & 80 & 120 & 160 & 200 & 240 & 280 & 320\end{array}\right]$} & {$\left[\begin{array}{lllllllll}0 & 0 & 0 & 0 & 0 & 0 & 0 & 0 & 0\end{array}\right]$} & {$\left[\begin{array}{lllllllll}0 & 0 & 0 & 0 & 0 & 0 & 0 & 0 & 0\end{array}\right]$} \\
\hline Head/neck 1, 2, 3 & {$\left[\begin{array}{lllllll}0 & 51.4 & 102.9 & 205.7 & 257.1 & 308.6\end{array}\right]$} & {$\left[\begin{array}{lllllll}0 & 0 & 0 & 0 & 0 & 0 & 0\end{array}\right]$} & {$\left[\begin{array}{lllllll}0 & 0 & 0 & 0 & 0 & 0 & 0\end{array}\right]$} \\
\hline
\end{tabular}

The effect of limiting the maximum intensity for each plan was evaluated by determining the total plan monitor units required for one fraction for both SMLC and DMLC delivery, the plan intensity map variation, and the clinical acceptability of the optimized plan. Also, the correlation coefficient was determined between intensity maps with varying maximum intensity ratios. Clinical acceptability was judged by individual plan dose volume histograms and specific dose metrics. For each case, the lowest maximum intensity ratio at which clinical acceptability was reached is reported, although we would expect this number to change slightly for each individual patient geometry. For the purposes of this study, we defined a clinically acceptable plan as one that met the planning constraints. The level to which a plan satisfied the planning objectives with respect to the unconstrained intensity plan was also evaluated.

\section{A. Brain}

For the brain test cases, four or five noncoplanar beams, originally placed by a dosimetrist, were used. The beam angle information for each of the cases is shown in Table I. The inverse plan objective function (shown in Table II) was designed using an in-house IRB approved protocol. According to our protocol, the inner target volume, PTV1, should receive $66 \mathrm{~Gy}$, with a minimum dose being $95 \%$ of the prescription dose with no more than $1 \%$ of PTV1 receiving greater than $105 \%$ of the prescription dose and no part of the volume receiving greater than $110 \%$ of the prescription dose. The outer target volume, PTV2, should receive $60 \mathrm{~Gy}$, with a minimum dose being $95 \%$ of the prescription dose. In addition, the volume of PTV2 that receives greater than $105 \%$ of the PTV1 prescription dose should be minimized, while still placing the priority on achieving the prescription dose in PTV1. For normal tissues, attempts are to be made to limit

TABLE II. Brain protocol objectives.

\begin{tabular}{ll}
\hline \hline \multicolumn{1}{c}{ Structure } & \multicolumn{1}{c}{ Objectives } \\
\hline PTV1 & $\begin{array}{l}\text { 66 Gy (minimum 95\%, maximum 105\%, and 1\% } \\
\text { of volume may receive up to 110\%) }\end{array}$ \\
PTV2 & $\begin{array}{l}\text { with PTV1 (minimum 95\%, maximum 105\% of PTV1 } \\
\text { wighe priority) }\end{array}$ \\
Right optic nerve & $\leqslant 60 \mathrm{~Gy}$ \\
Left optic nerve & $\leqslant 60 \mathrm{~Gy}$ \\
Chiasm & $\leqslant 60 \mathrm{~Gy}$ \\
Brainstem & $\leqslant 65 \mathrm{~Gy}$ \\
\hline \hline
\end{tabular}

the dose to the optic nerves and chiasm to less than $60 \mathrm{~Gy}$, and the dose to the brainstem to less than $65 \mathrm{~Gy}$.

\section{B. Prostate}

For the prostate test cases, we used nine beams at $6 \mathrm{MV}$ based on work by Pirzkall et $a l^{41}$ They found that nine beams were necessary at $6 \mathrm{MV}$ to avoid increased doses to normal tissue distant from the PTV. Similar to the Pirzkall work, we applied dose volume objectives according to RTOG $9406 .{ }^{42}$ However, instead of defining only the prostate as the PTV, we used the prostate target volumes employed routinely in our clinic, which include the prostate with a 5 $\mathrm{mm}$ expansion to receive full dose (75.6 Gy for this study) and an additional $5 \mathrm{~mm}$ margin that receives $54 \mathrm{~Gy}$. The margin is used because prostate patients in our clinic are imaged and aligned daily with implanted fiducial markers. ${ }^{43}$ The beam angle information and target and normal tissue objectives are given in Tables I and III, respectively. In addition to those objectives listed, unnecessary dose to uninvolved normal tissues is to be minimized, and maximum dose shall not exceed that of the target volumes.

\section{Head/neck}

For the head/neck geometry, the objective function was designed using our in-house head/neck IMRT protocol, which has multiple target prescriptions (see Table IV). The optimization constraints on the targets require that mean target doses shall be $100 \% \pm 3 \%$ of the prescribed dose, the minimum target doses shall be greater than or equal to $93 \%$ of the prescribed dose, and the maximum hot spot in the target shall be less than or equal to $115 \%$ of the prescribed dose (delivered to a volume of at least $0.5 \mathrm{cc}$ ). The maximum dose outside the targets should be less than $105 \%$ of the

TABLE III. Prostate IMRT planning objectives.

\begin{tabular}{ll}
\hline \multicolumn{1}{c}{ Structure } & \multicolumn{1}{c}{ Objectives } \\
\hline Prostate $+5 \mathrm{~mm}$ & $75.6 \mathrm{~Gy}$ (minimum 95\%, maximum 110\%) \\
Prostate $+1 \mathrm{~cm}$ & $54 \mathrm{~Gy}$ (minimum 95\%, maximum 110\% of prostate \\
& $+5 \mathrm{~mm}$ ) \\
Rectum & $\leqslant 10 \%$ volume receives $>56 \mathrm{~Gy}$ \\
Bladder & $\leqslant 20 \%$ volume receives $>60 \mathrm{~Gy}$ \\
Right femur & $\leqslant 10 \%$ volume receives $>40 \mathrm{~Gy}$ (maximum $45 \mathrm{~Gy}$ ) \\
Left femur & $\leqslant 10 \%$ volume receives $>40 \mathrm{~Gy}$ (maximum $45 \mathrm{~Gy}$ ) \\
Penile bulb & $\leqslant 40 \%$ volume receives $>50 \mathrm{~Gy}$ \\
Seminal vesicles & $\leqslant 20 \%$ volume receives $>40 \mathrm{~Gy}$ \\
\hline \hline
\end{tabular}


TABLE IV. Head/neck IMRT protocol planning objectives.

\begin{tabular}{ll}
\hline \hline \multicolumn{1}{c}{ Structure } & \multicolumn{1}{c}{ Objectives } \\
\hline PTV1 & $70 \mathrm{~Gy}($ mean $\pm 3 \%$, minimum 93\%, maximum 115\%) \\
PTV2 & $60 \mathrm{~Gy}($ mean $\pm 3 \%$, minimum 93\%, maximum 115\%) \\
Nodal boost PTV & $70 \mathrm{~Gy}($ mean $\pm 3 \%$, minimum 93\%, maximum 115\%) \\
High risk nodal PTV & $64 \mathrm{~Gy}($ mean $\pm 3 \%$, minimum 93\%, maximum 115\%) \\
Low risk nodal PTV & $57.6 \mathrm{~Gy}($ mean $\pm 3 \%$, minimum 93\%, maximum 115\%) \\
Spinal cord & $\leqslant 45 \mathrm{~Gy}$ \\
Spinal cord $+5 \mathrm{~mm}$ & $\leqslant 50 \mathrm{~Gy}$ \\
Brainstem & $\leqslant 54 \mathrm{~Gy}$ \\
Right parotid & Mean dose $\leqslant 26 \mathrm{~Gy}$ \\
Left parotid & Mean dose $\leqslant 26 \mathrm{~Gy}$ \\
Mandible & $\leqslant 70 \mathrm{~Gy}$ \\
Submandibulars & Minimize dose \\
Oral cavity & Less than or equal to $70 \mathrm{~Gy}$ \\
\hline \hline
\end{tabular}

prescribed dose to PTV1. Normal tissue constraints are given in Table IV for the spinal cord, brainstem, mandible, and uninvolved oral cavity. Other objectives include achieving a mean dose in at least one parotid gland of less than $26 \mathrm{~Gy}$, and minimizing dose to the uninvolved submandibular glands and all other uninvolved normal tissue. In cases where normal structures such as the mandible and oral cavity overlap target volumes, the strict maximum dose constraints on those structures may be relaxed to achieve target dose.

\section{RESULTS}

\section{A. Brain}

By employing maximum intensity limits in the brain, it was possible to achieve clinical acceptability with a substantial decrease in plan intensity map variation and monitor units when compared to the unconstrained intensity plans. Table $\mathrm{V}$ summarizes the reductions that were possible in each of the cases tested. Both brain 1 and brain 3 had similar geometries, while the targets in brain 2 had large areas of overlap with parts of the brainstem and optic chiasm. Dose volume histograms for PTV1, PTV2, the right and left optic nerves, chiasm, and brainstem for brain 1 are displayed for maximum ratios of $1.15,1.75$, and 3.62 (unconstrained in- tensity plan) in Fig. 1(a). The corresponding dose distributions for an axial cut are shown in Fig. 3(b) for both the first acceptable plan and the unconstrained intensity plan. In all cases above a maximum intensity ratio of 1.15 , coverage of PTV1 and PTV2 was identical, and all normal tissue constraints were met. The unconstrained intensity plan did allow for slight improvements in normal tissue mean and maximum doses, which can be seen in Fig. 1(b). Here we see a spreading out of the low dose regions into the normal brain for MIR=1.15. Shown in the top plot of Fig. 2, the maximum dose metrics for the right optic nerve, chiasm, and brainstem stay constant due to the maximum dose objectives in those structures, however, we do see slight decreases in the maximum dose to the left optic nerve. The mean doses for all normal structures are reduced slightly as the maximum intensity is increased, however, these values tend to converge as the plan reaches higher values of the MIR. The largest improvements were seen in the mean doses of the optic chiasm and brainstem, which had dose reductions of 5.0 and $7.3 \mathrm{~Gy}$, respectively, when not applying intensity limits. Monitor units (MU) were significantly higher in the unconstrained intensity plan (758 MU) compared to that of acceptable plans with constrained intensities (starting at 473 $\mathrm{MU}$ ), and the possible reduction in the plan intensity map

TABLE V. Brain, prostate, and head/neck case reductions as compared to the unconstrained intensity plans (\%).

\begin{tabular}{|c|c|c|c|c|c|c|}
\hline \multirow[b]{2}{*}{ Case } & \multirow[b]{2}{*}{ MIR (unconstrained) } & \multirow[b]{2}{*}{ MIR (first accepted plan) } & \multirow[b]{2}{*}{$C_{\text {uncon,con }}(1$ s.d. $)$} & \multirow[b]{2}{*}{ PIMV \% reductions } & \multicolumn{2}{|c|}{ MU \% reductions } \\
\hline & & & & & SMLC & DMLC \\
\hline Brain 1 & 3.62 & 1.15 & $0.80(0.05)$ & 30.0 & 37.6 & 28.8 \\
\hline Brain 2 & 2.41 & 1.15 & $0.84(0.09)$ & 18.2 & 19.0 & 11.4 \\
\hline Brain 3 & 2.64 & 1.15 & $0.84(0.07)$ & 25.5 & 32.6 & 24.2 \\
\hline Prostate 1 & 5.07 & 1.45 & $0.69(0.14)$ & 34.0 & 30.0 & 14.0 \\
\hline Prostate 2 & 3.87 & 1.60 & $0.71(0.08)$ & 27.0 & 24.8 & 9.6 \\
\hline Prostate 3 & 4.10 & 1.60 & $0.71(0.10)$ & 22.5 & 22.1 & 0.2 \\
\hline HN 1 & 3.71 & 1.45 & $0.85(0.05)$ & 11.7 & 23.8 & 16.8 \\
\hline HN 2 & 3.00 & 1.15 & $0.81(0.03)$ & 31.8 & 26.7 & 24.2 \\
\hline HN 3 & 1.90 & 1.15 & $0.93(0.02)$ & 15.3 & 10.8 & 9.9 \\
\hline
\end{tabular}



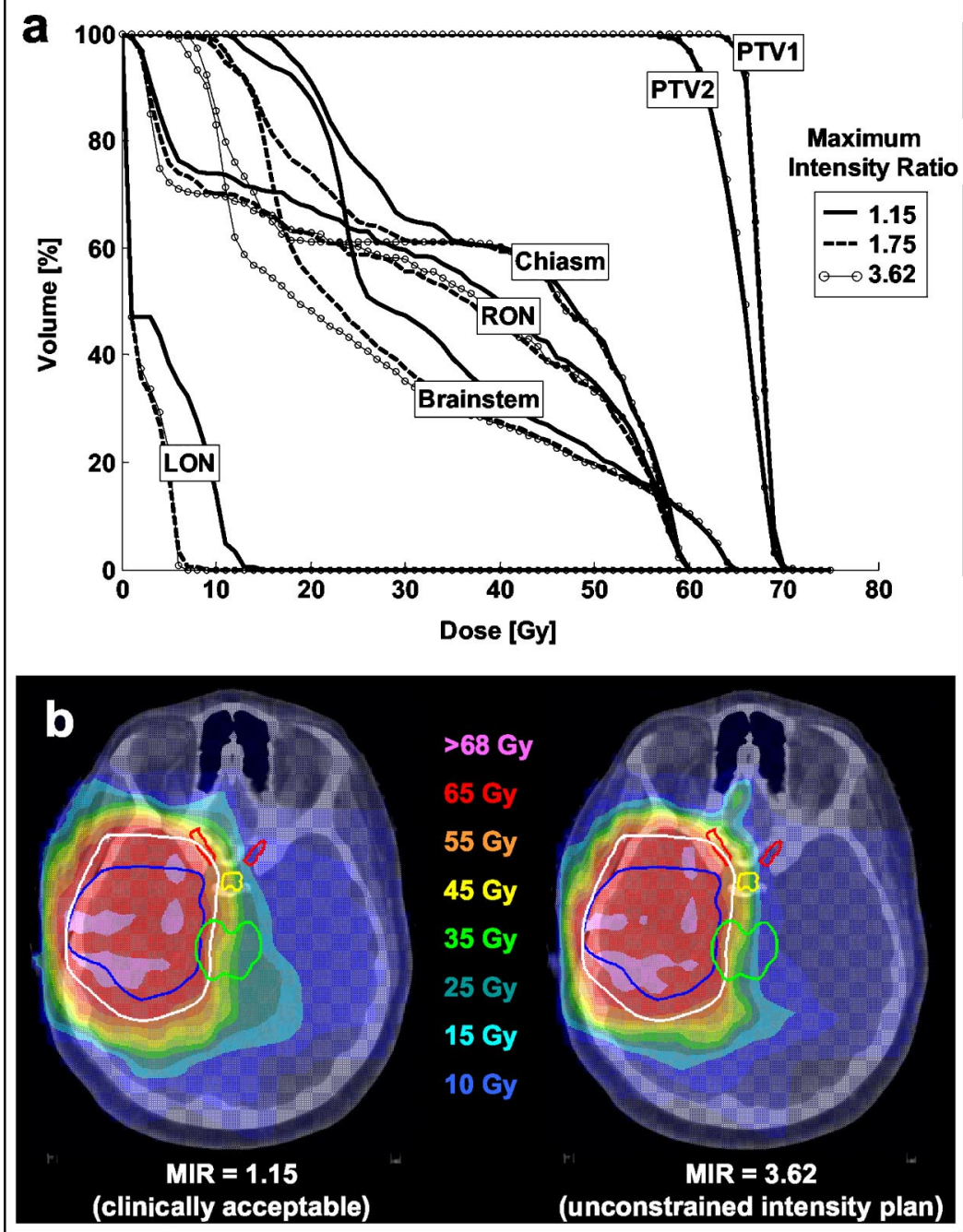

FIG. 1. Brain case-(a) DVHs of PTV1, PTV2, both optic nerves, chiasm, and brainstem for IMRT plans with maximum intensity limits (MIR $=1.15,1.75,3.62$ ). Each plan satisfied protocol target and normal tissue limits. (b) Axial dose distributions for the first acceptable plan (left) and the unconstrained intensity plan (right). Structure contours shown are PTV1 (blue), PTV2 (white), optic nerves (red), optic chiasm (yellow), and brainstem (green).

variation was $30.0 \%$. A plot of the MU required for one fraction of SMLC delivery as a function of maximum intensity ratio is shown in the bottom plot of Fig. 2. The average correlation coefficient of the beams compared to the unconstrained intensity plan is also shown. As the maximum intensity ratio increases, we see the correlation coefficient of the beams begin to converge while the MU continue to increase. Similar trends were seen in brain 2 and brain 3, with the improvements varying slightly for different normal tissues depending on the tumor location. An acceptable plan was achieved with only an $80 \%$ average correlation coefficient as compared to the unconstrained intensity plan beams (for the example shown). Values of the correlation coefficients between the first acceptable plans and the unconstrained intensity plans are shown in Table V.

\section{B. Prostate}

When using maximum intensity constraints in the prostate, it was possible to reduce the number of monitor units and intensity map variation, while still meeting each of the planning constraints. The possible reductions in plan intensity map variation, SMLC monitor units, and DMLC monitor units are given in Table $\mathrm{V}$ along with the average correlation coefficients between the unconstrained intensity plans and the acceptable plans with the lowest MIR values. The mean and standard deviation of the reduction in MU for SMLC when compared to DMLC for all inverse plans are also shown in Table V. For prostate 1, when compared to the first clinically acceptable plan, reductions in mean dose of $6.5 \mathrm{~Gy}$ in the rectum and 2.6 Gy in the bladder were possible when removing intensity constraints, but monitor units per fraction were increased from 847 to 1211 MU. Dose-volume histograms are shown in Fig. 3(a) for the prostate $+5 \mathrm{~mm}$, prostate $+1 \mathrm{~cm}$, rectum, and bladder at various maximum intensity ratios. At each maximum intensity ratio shown, all planning constraints are satisfied, although some improvement in overall minimization of normal tissue dose is observed in the unconstrained intensity plan. Figure 3(b) shows the optimized dose distributions for the first acceptable plan and the unconstrained plan. We see a different trend in the dose deposition between the two cases, with the majority of entrance/exit dose in the anterior/posterior plane in the intensity limited plan as compared to the unconstrained intensity plan which appears to rely less on the AP 


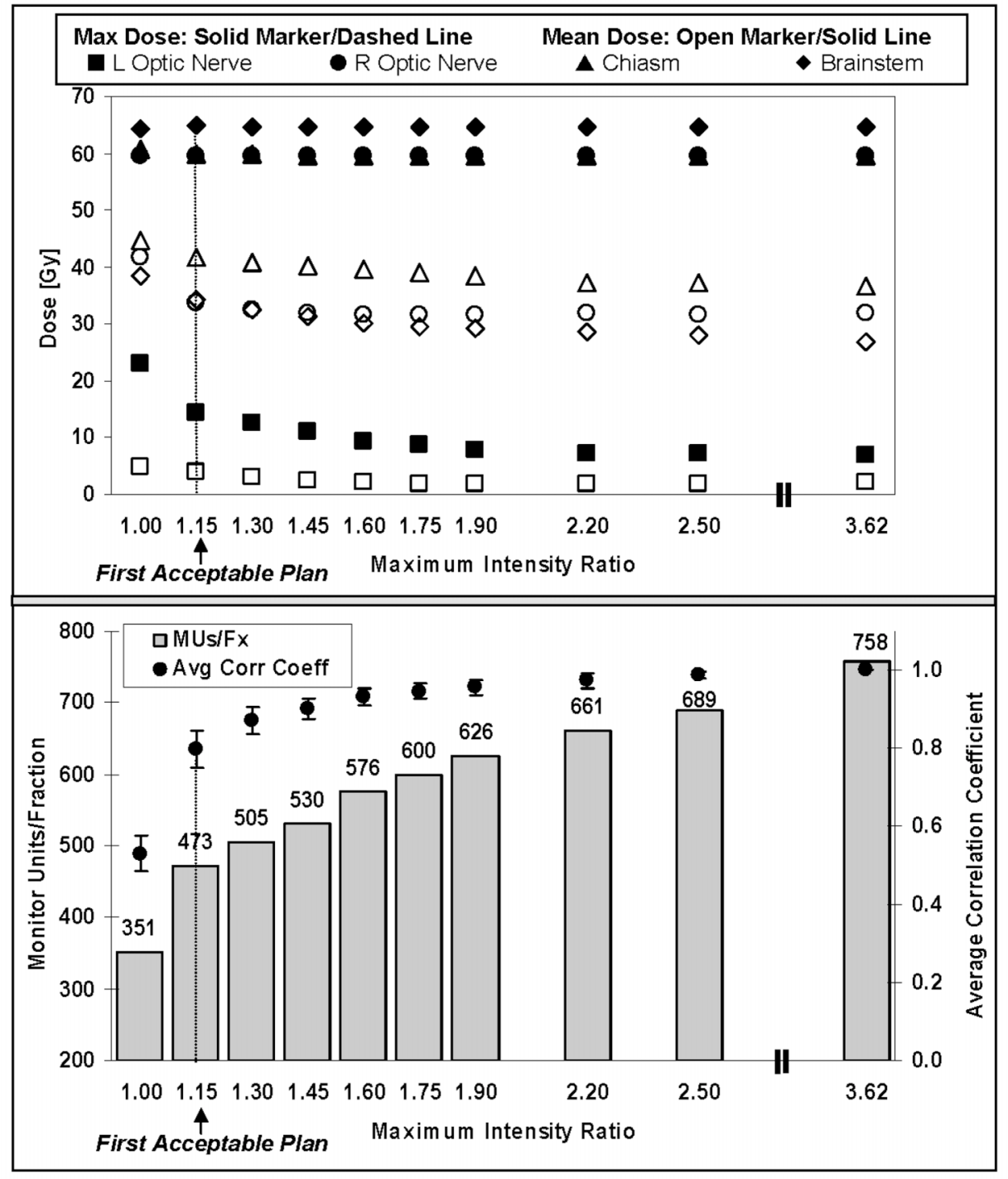

FIG. 2. Brain case-top plot: Normal tissue mean and maximum doses as a function of increasing maximum intensity ratio. Bottom plot: Monitor units per fraction (left), and average correlation coefficient of the fields (right), as a function of increasing maximum intensity ratio. The first clinically acceptable plan is observed at $\mathrm{MIR}=1.15$.

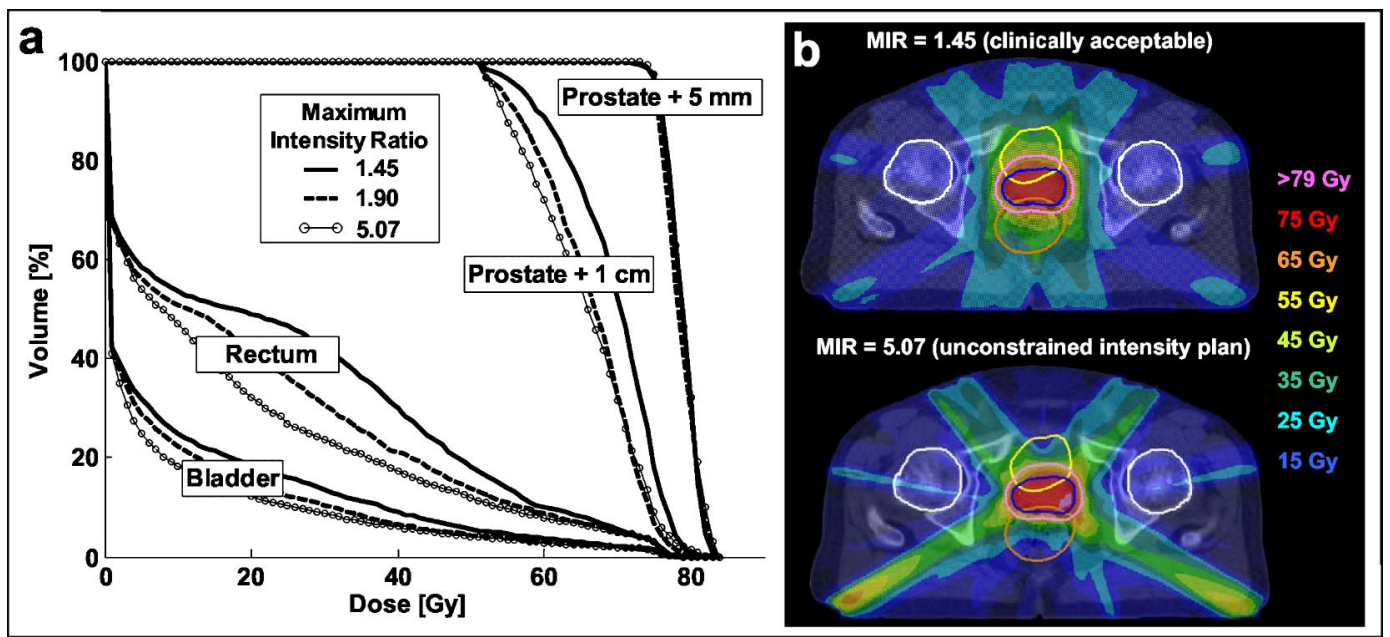

FIG. 3. Prostate case-(a) DVHs of the prostate $+5 \mathrm{~mm}$, prostate $+1 \mathrm{~cm}$, the rectum, and the bladder for IMRT plans with maximum intensity limits and an unconstrained intensity plan (MIR=5.07). Each plan shown satisfies both target and normal tissue constraints and dose-volume objectives except for MIR =1.30. (b) Axial dose distributions for the first acceptable plan (top) and the unconstrained intensity plan (bottom). Structure contours shown are prostate $+5 \mathrm{~mm}$ (blue) prostate $+1 \mathrm{~cm}$ (violet), rectum (brown), bladder (yellow), and femurs (white). 


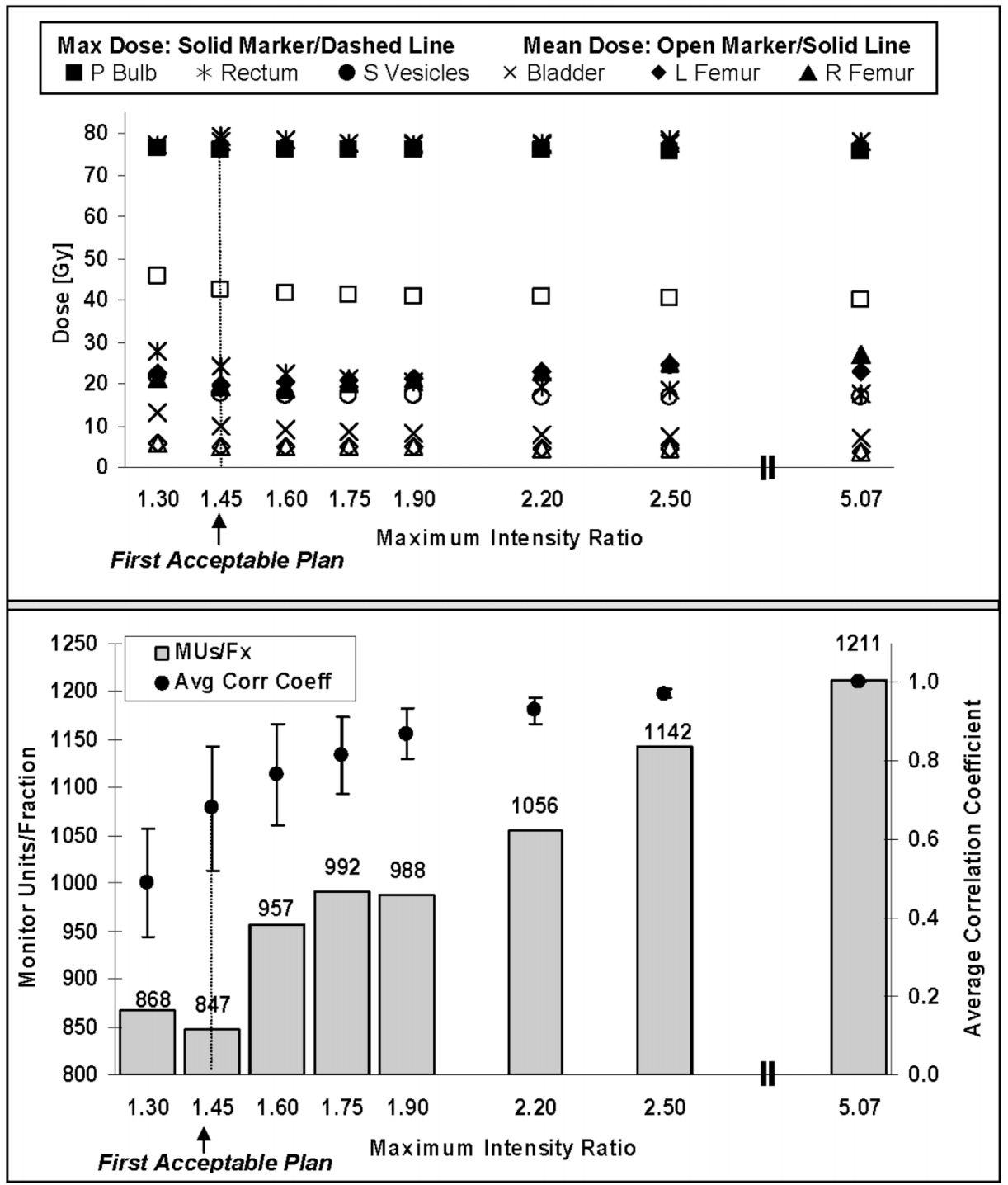

FIG. 4. Prostate case- - top plot: Maximum and mean normal tissue doses as a function of increasing maximum intensity ratio. Bottom plot: Monitor units per fraction (left), and average correlation coefficient of the fields (right), as a function of increasing maximum intensity ratio. The first clinically acceptable plan is observed at $\mathrm{MIR}=1.45$. and PA beams. Figure 4 shows considerable increases in MU/fraction, with only slight changes in the normal tissue doses. The top plot in Fig. 4 shows that the maximum and mean normal tissue metrics stay fairly constant after the first acceptable plan is reached. The only obvious changes are increases in the maximum dose delivered to the femurs corresponding to very slight reductions in mean dose to the rectum and bladder. In the bottom plot of Fig. 4, the correlation coefficient converges to one as the MIR reaches its maximum value for the unconstrained intensity plan. However, as shown in Table $\mathrm{V}$, we reach an acceptable plan at only a $69 \%$ average correlation with the unconstrained intensity beams and are able to reduce the PIMV by $34 \%$.

\section{Head/neck}

In the studied head/neck cases, it was possible to achieve reductions in SMLC and DMLC monitor units per fraction when using maximum intensity limits during IMRT plan- ning. Table $\mathrm{V}$ shows the size of these reductions, the maximum intensity ratio of the first acceptable plans in each case, and the average correlation of the beams in these plans versus the unconstrained intensity plans. It is of interest to point out that HN 3 had large bilateral boost volumes, possibly affecting the number of degrees of freedom needed to further reduce plan complexity. For each case, all planning objectives, including the sparing of the contralateral parotid, were met. In HN 1, considerable modulation was still necessary due to the complex geometry, only leading to a $12 \%$ reduction in plan intensity map variation when using maximum intensity limits. When not limiting the maximum intensity, slight reductions in the ipsilateral parotid mean doses were achieved, although it was not possible to spare this parotid due to its position relative to the target volumes. Normal tissue dose metrics for HN 1 are shown in Fig. 5 at varying levels of maximum intensity ratio. In Figs. 6(a) and 6(b), dose-volume histograms (DVHs) are displayed for all targets and several normal structures. DVHs in the target volumes are almost identical, while there are visible changes in the 


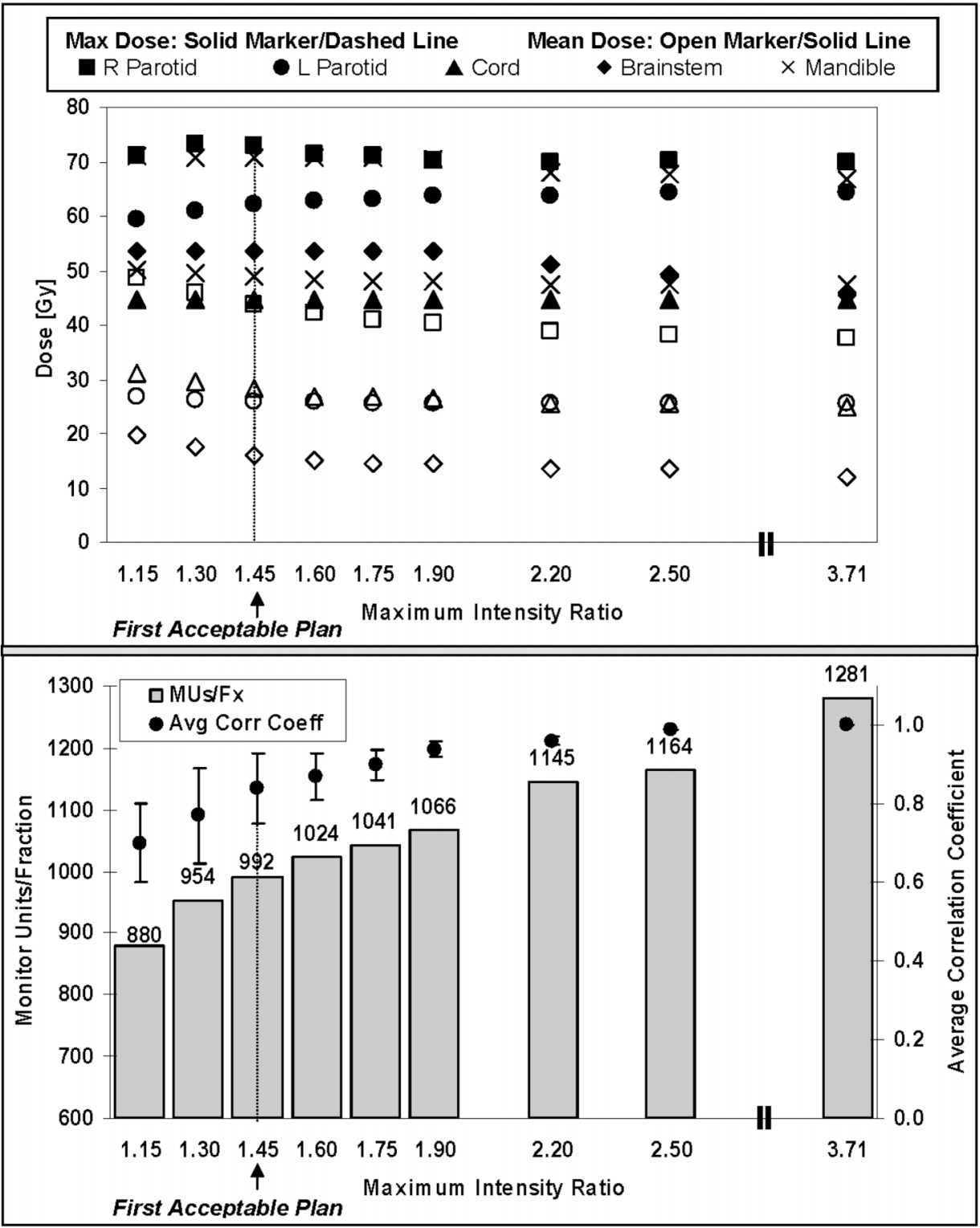

FIG. 5. Head/neck case-top plot: Mean and maximum normal tissue doses as a function of increasing maximum intensity ratio. Mean doses are solid lines and maximum doses are dashed lines. Bottom plot: Monitor units per fraction (left), and average correlation coefficient of the fields (right), as a function of increasing maximum intensity ratio. The first clinically acceptable plan is observed at $\mathrm{MIR}=1.30$. normal structure DVHs-most noticeably in the brainstem. In Fig. 6(c), dose distributions are seen for the first acceptable plan and the unconstrained intensity plan. Both distributions are similar with only slight changes in the dose deposition patterns. We can see from the top plot of Fig. 5 that the normal tissue mean and maximum doses are fairly constant after the first acceptable plan is met. However, there are still slight changes that can be observed in the mean doses to the mandible, brainstem, and left parotid and maximum doses to the right parotid and brainstem as the maximum intensity limit is increased. The bottom plot in Fig. 5 displays the increased monitor units necessary to improve the normal tissue DVHs as seen in Fig. 6. Again, we see that the correlation coefficient of the beams relative to the unconstrained intensity plan converges to one while the monitor units continue to increase steadily, especially when the maximum intensity is not limited. The first acceptable plan was reached at an average correlation of 0.85 with the unconstrained in- tensity plan. The difference in the intensity maps as the maximum intensity ratio increases for a typical beam is shown in Fig. 7. For each intensity map, the correlation coefficient with respect to the unconstrained intensity plan is shown along with the number of monitor units. Note the large increase in monitor units for the unconstrained intensity beam compared to the other plans. We observe that nearly all beamlets are at the maximum allowed value at $\mathrm{MIR}=0.85$. As the MIR increases, we continue to see changes in the beamlet pattern. As we reach MIRs past 1.45, only slight changes in the high intensity regions of the beam can be clearly observed. We can see an isolated peak appears toward the upper left of the field as well as a large region of high intensity in the upper right of the field. These features, as can be seen in the DVHs and dose metrics, do not affect the clinical acceptability of the plan, but do increase the MU significantly. 


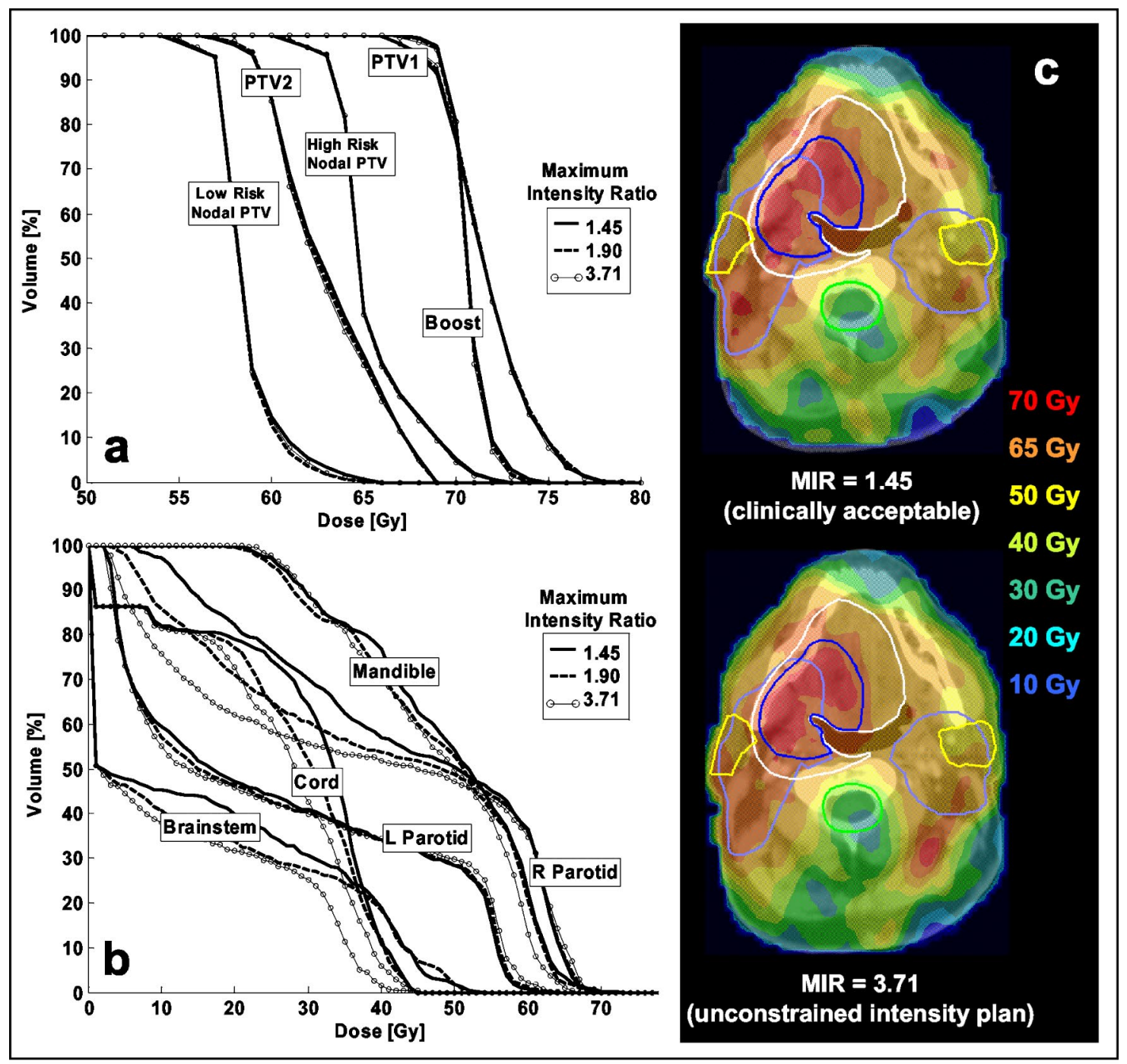

FIG. 6. Head/neck case-(a) DVHs of the five target volumes for IMRT plans with maximum intensity limits and an unconstrained intensity plan (MIR =3.71), (b) DVHs of the parotids, cord, brainstem, and mandible, and (c) axial dose distributions for the first acceptable plan (top) and the unconstrained intensity plan (bottom). Structure contours shown are PTV1 (blue), PTV2 (white), parotids (yellow), cord +3 mm (green), and nodal target volumes (light blue).

\section{DISCUSSION}

Mohan et al. have evaluated many of the difficulties that arise when dealing with complex intensity patterns, and have motivated the reduction of unnecessary modulation in IMRT beams. ${ }^{10}$ We have proposed maximum intensity limits during inverse planning as a possible approach to dealing with this problem. For several clinical sites, the dosimetric advantages and disadvantages of limiting the maximum beam intensity were evaluated by optimizing each case at different values of the maximum intensity ratio, starting at 0.85 up to the maximum value for the unconstrained intensity plan. In each case, it was possible to achieve clinical acceptability with substantial decreases in beam modulation and monitor units when applying maximum intensity constraints. Monitor unit reductions with SMLC delivery up to $38 \%, 30 \%$, and $27 \%$ were seen in the brain, prostate, and head/neck, respectively. Corresponding decreases for DMLC delivery of up to $29 \%$, $14 \%$, and $24 \%$ were also observed. Also, the plan intensity map variation was reduced by up to $30 \%, 34 \%$, and $32 \%$ in the brain, prostate, and head/neck. In the head/neck, a somewhat larger degree of beam modulation was necessary and desirable to reach the planning objectives, and in two of the three cases in this site, reductions of only $12 \%$ and $15 \%$ in plan intensity map variation in the constrained intensity plan demonstrate that this method is capable of preserving necessary beam modulation while still meeting the specified plan objectives. As discussed previously, one of the head/neck cases (HN 3) had a very complex geometry with bilateral nodal boost volumes, making it difficult to spare normal structures. However, with the maximum beamlet intensity limit, it was still possible to reduce SMLC and DMLC MU by $11 \%$ and $10 \%$ while meeting critical target coverage and normal tissue limits.

In similar situations, some smoothing algorithms may be ineffective at reducing $\mathrm{MU}$ without compromising coverage or sparing due to the sensitive geometry. For example, Sun et 

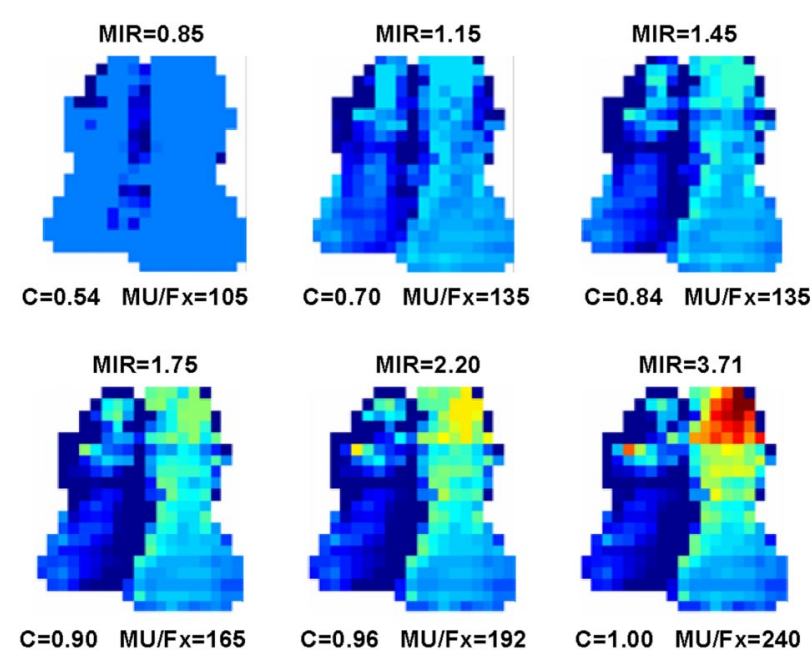

FIG. 7. Head/neck case-beam intensity patterns for maximum intensity ratios $0.85-3.71$. Shown below each intensity map is the correlation coefficient with respect to the unconstrained intensity plan $(\mathrm{MIR}=3.71)$, and the monitor units per fraction required to deliver the beam via SMLC delivery.

al. introduced a smoothing procedure based on the structure index followed by re-optimization of segment weights to improve delivery efficiency. This method was not as effective in reducing $\mathrm{MU}$ in a relatively uncomplicated head/neck example having a main GTV and CTV and four normal tissue objectives. ${ }^{25}$ For more complicated cases, such as the head/ neck examples used in the current work, the structure indexbased smoothing may be even less effective due to the numerous regions of interest and overlap of structures. Smoothing algorithms that are applied post-optimization generally smooth everywhere in the field, therefore they cannot distinguish between desirable gradients and undesirable ones, usually resulting in a degradation of the plan according to the objective function. When smoothing is part of the objective function, it can be difficult to quantify the direct tradeoffs that must be made between plan objectives and the smoothness criteria. ${ }^{10,13}$ However, promising results have been shown for plans of average complexity by introducing smoothness criteria into the cost function at the expense of decreased delivery efficiency, when compared to methods that smooth outside of the cost function. ${ }^{13}$

In the current work with intensity limits, the tradeoff to achieve increased delivery efficiency is generally an increase in dose to some of the normal tissues. Examination of the DVHs in Figs. 1, 3, and 6 shows that the high priority objectives are met when using the intensity limits, and many of the differences seen when compared to unconstrained plans are only in the low dose regions. While it is always preferred to decrease dose wherever possible, the clinical importance of these changes in the low dose region is difficult to judge. Considering the additional transmission and leakage dose delivered to the patient during more modulated and complex deliveries, the advantages of using unconstrained intensity limits could be diminished, or more important, outweighed, by the increased normal tissue dose due to leakage/ transmission. The average transmission is machine depen- dent and is approximately $2 \%$ of the total monitor units for our linear accelerator and MLC design (Varian, $2100 \mathrm{EX}$, Millennium MLC, Palo Alto, CA). ${ }^{44}$ In the cases we have shown, these transmission rates result in an average increase in dose from the constrained to unconstrained plans of 4.0 cGy (1.7 s.d.) for the static deliveries and 3.4 cGy (2.3 s.d.) for the dynamic deliveries. As Mohan et al. suggest, in complex deliveries it is not uncommon for some points to receive $100 \%$ of their dose through indirect means. ${ }^{10}$ Thus, the extra effort to reduce normal tissue doses by making minor intensity adjustments that increase the total monitor units are likely unproductive, and may even increase the total doses eventually received when more accurately accounting for transmission and leakage and geometric uncertainties. Hall and $\mathrm{Wu}$ have suggested that this increased leakage radiation may contribute to an increased risk of second malignancies, and a joint publication by the American Society for Therapeutic Radiation Oncology (ASTRO) and the American Association of Physicists in Medicine (AAPM) has also pointed out the compromises that must be made when considering the increases in MU frequently seen in clinical IMRT. ${ }^{14,15}$

Table V and Figs. 2, 4, and 5 show large increases in the number of monitor units required for the unconstrained intensity plans as compared to several of the constrained intensity plans. Many of these increases are due to isolated large intensity "spikes" in the optimized beamlet distributions. These peaks in the intensity pattern can result from artifacts in the point-based optimization schemes used in inverse planning and may not be necessary to produce a plan of high quality. We can also observe in Figs. 2, 4, and 5 that most normal tissue dose metrics reach a point where they begin to stay fairly constant as a function of increasing maximum intensity ratio. At this point, the correlation coefficients also begin to converge to one. This may be due to the fact that the optimization has reached the maximum intensity ratio at which all important priorities are met and further increasing the maximum intensity allows the system to produce small fluctuations in neighboring beamlets or large changes in single beamlets that only slightly affect the objective value. At this point, it may no longer be necessary to allow increases in the maximum beamlet intensity because the main result is an increase in MU. However, it is important to point out that it is not essential for the beamlet patterns to correlate with the unconstrained beamlet patterns to a very high degree. By limiting the maximum intensity, the solution space of the problem is altered so that an equally acceptable solution could be achieved without a high degree of correlation. Looking at all beams in the first acceptable plans for each treatment site, we have an average correlation coefficient of $82.6 \%(6.9 \%)$ for brain and an average of $86.3 \%(6.5 \%)$ for head/neck. The prostate correlation coefficient average is even lower at $70.5 \%(10.7 \%)$, which may be due partly to the fact that the prostate plans had $0.5 \mathrm{~cm}$ by $0.5 \mathrm{~cm}$ beamlets as compared to $1 \mathrm{~cm}$ by $1 \mathrm{~cm}$ beamlets. Another factor is that different tradeoffs were made in the cost function as can be seen from the different shapes of the dose distributions for prostate 1 in Fig. 3(b). 
It should be noted that using an intensity limit within the optimization search (or other constraints on beamlet intensities) is different than applying that same kind of limit within the leaf sequencing operation. Since the sequencer is typically independent of the optimization process, applying these limits during sequencing ignores any clinical tradeoffs that are made according to the cost function. Instead, the leaf sequencer tries to achieve a sequence that matches the input (planned) intensities, so any deviations from the planned intensity are only evaluated on an intensity basis and are not related to the actual clinical compromise that may result from a deviation between the planned and deliverable intensity map. With the delivery-related limitations (beamlet maximum intensity, in this case) within the plan optimization search, the tradeoffs are made using the clinical cost function resulting in clinically relevant compromises. The same holds true when incorporating smoothing and hardware delivery constraints into the optimization process. ${ }^{12,16-18}$ However, incorporation of delivery constraints during optimization does not discriminate against unnecessary modulation and excessive MU. Siebers et al. have shown that there is a possibility of reducing the MU significantly by incorporating leaf sequencing into the optimization process, although much of the MU reduction may have been due to intensity filtering and smoothing in the leaf-sequencing algorithm. ${ }^{12}$

Another competing technique would be to use discrete intensity levels for the optimization. ${ }^{45,46}$ This method would be able to remove large intensity peaks in a similar way to applying intensity limits if the highest intensity level was set to a reasonable value. In addition, discretizing the intensity levels would remove any small fluctuations between neighboring beamlets that also contribute to increases in MU. One drawback to using predefined intensities is that the degrees of freedom are diminished for complicated cases, possibly preventing some objectives to be met. In these cases, it may be possible to increase the number of intensity levels through an iterative process without significantly affecting planning time. Gains in efficiency are also possible with more advanced leaf sequencing algorithms, leading to less discrepancy between planned and delivered fields. ${ }^{17-21}$ However, this technique is not meant to remove undesirable highfrequency components from the planned intensity fields, which could possibly make the plan more vulnerable to slight geometric changes. Although a study of geometric sensitivity was not performed in this work, it may follow from the reductions seen in the plan intensity map variation values that the intensity limited plans would be somewhat less sensitive to small shifts in the patient geometry than unconstrained IMRT plans.

Due to the advantages in decreasing monitor units and hence leakage dose when applying maximum intensity limits, we have modified our clinical IMRT planning to incorporate this approach when appropriate. At this time for each patient, an initial patient plan is optimized with unconstrained intensity to determine clinical compromises that are due solely to the objective functions for the tumor and normal tissues. The resulting plan is reviewed by the physician to determine the acceptability of the plan. Then, a dosim- etrist, based on experience and the plan information such as the prescription dose and number of beam angles, will choose a maximum intensity to use in reoptimizing the patient plan. If the resulting plan is acceptable to the physician and has a significant decrease in MU after sequencing, the constrained plan is generally accepted. While the current process is iterative, it can typically be performed in $20 \mathrm{~min}$ or less. Applying a limit to the maximum beamlet is straightforward in our in-house optimization system. However, limiting this parameter may not be possible at this time in other optimization systems. In that case, other approaches may be used to obtain similar results including methods to decrease the number of intensity levels as discussed earlier.

It is clear that the maximum intensity limits described here or smoothing functions used on the beamlet distribution during optimization only address a limited aspect of the general problem, which is to decrease complexity-unless that complexity is really needed to achieve the desired clinical result. Other approaches, such as incorporating more advanced delivery objectives into optimization, moving away from point-based and beamlet-based optimization, and improved tradeoff evaluation tools may be necessary to make further advances in this area. ${ }^{11,12}$

\section{CONCLUSIONS}

Incorporating intensity limits into the optimization process is a simple and effective way to reduce unnecessary beam modulation and monitor units required for delivery in IMRT plans. In the brain, prostate, and head/neck, monitor unit reductions up to $38 \%, 30 \%$, and $29 \%$ as compared to unconstrained intensity plans were possible while still meeting planning objectives. The intensity map variation was reduced by up to $30 \%$ and $34 \%$ in the brain and prostate, while the complex head/neck variation was able to be preserved when necessary to meet the planning objectives. The dosimetric advantage of not constraining the maximum intensity is an overall minimization of normal tissue dose, however this effect may be offset by increased transmission and leakage with greater numbers of monitor units being required for delivery.

\section{ACKNOWLEDGMENT}

This work was supported in part by NIH P01-CA59827.

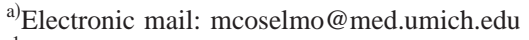

${ }^{1}$ C. Hurkmans, B. Cho, E. Damen, L. Zijp, and B. Minjnheer, "Reduction of cardiac and lung complication probabilities after breast irradiation using conformal radiotherapy with or without intensity modulation," Radiother. Oncol. 62, 163-171 (2002).

${ }^{2}$ N. Dogan, S. King, B. Emami, N. Mohideen, N. Mirkovic, B. Leybovich, and A. Sethi, "Assessment of different IMRT boost delivery methods on target coverage and normal tissue sparing," Int. J. Radiat. Oncol., Biol., Phys. 57, 1480-1491 (2003).

${ }^{3}$ A. Pirzkall, M. Carol, F. Lohr, A. Höss, M. Wannenmacher, and J. Debus, "Comparison of intensity-modulated radiotherapy with conventional conformal radiotherapy for complex-shaped tumors," Int. J. Radiat. Oncol., Biol., Phys. 48, 1371-1380 (2000).

${ }^{4} \mathrm{~V}$. Wu, D. Kwong, and J. Sham, "Target dose conformity in 3-dimensional conformal radiotherapy and intensity modulated radiotherapy," Radiother. Oncol. 71, 201-206 (2004).
} 
${ }^{5}$ C.-Y. Hsiung, E. Yorke, C.-S. Chui, M. Hung, C. Ling, E.-Y. Huang, C.-J Wang, H.-C. Chen, S.-A. Yeh, H.-C. Hsu, and H. Amols, "Intensitymodulated radiotherapy versus conventional three-dimensional conformal radiotherapy for boost or salvage treatment of nasopharyngeal carcinoma,". Int. J. Radiat. Oncol., Biol., Phys. 53, 638-647 (2002).

${ }^{6}$ M. Kam, R. Chau, J. Suen, P. Choi, and P. Teo, "Intensity-modulated radiotherapy in nasopharyngeal carcinoma: Dosimetric advantage over conventional plans and feasibility of dose escalation," Int. J. Radiat. Oncol., Biol., Phys. 56, 145-157 (2003).

${ }^{7}$ H. Murshed, H. Liu, Z. Liao, J. Barker, X. Wang, S. Tucker, A. Chandra, T. Guerrero, C. Stevens, J. Change, M. Jeter, J. Cox, R. Komaki, and R. Mohan, "Dose and volume reduction for normal lung using intensitymodulated radiotherapy for advanced-stage non-small-cell lung cancer," Int. J. Radiat. Oncol., Biol., Phys. 58, 1258-1267 (2004).

${ }^{8}$ G. Luxton, S. Hancock, and A. Boyer, "Dosimetry and radiobiologic model comparison of IMRT and 3D conformal radiotherapy in the treatment of carcinoma of the prostate," Int. J. Radiat. Oncol., Biol., Phys. 59, 267-284 (2004).

${ }^{9}$ D. Huang, P. Xia, P. Akazawa, C. Akazawa, J. Quivey, L. Verhey, M. Kaplan, and N. Lee, "Comparison of treatment plans using intensitymodulated radiotherapy and three-dimensional conformal radiotherapy for paranasal sinus carcinoma," Int. J. Radiat. Oncol., Biol., Phys. 56, 158-168 (2003).

${ }^{10}$ R. Mohan, M. Arnfield, S. Tong, W. Wu, and J. Siebers, "The impact of fluctuations in intensity patterns on the number of monitor units and the quality and accuracy of intensity modulated radiotherapy," Med. Phys. 27, 1226-1237 (2000).

${ }^{11}$ J. Markman, D. Low, A. Beavis, and J. Deasy, "Beyond bixels: Generalizing the optimization parameters for intensity modulated radiation therapy," Med. Phys. 29, 2298-2304 (2002).

${ }^{12}$ J. Siebers, M. Lauterbach, P. Keall, and R. Mohan, "Incorporating multileaf collimator leaf sequencing into iterative IMRT optimization," Med Phys. 29, 952-959 (2002).

${ }^{13}$ S. Spirou, N. Fournier-Bidoz, J. Yang, C. Chui, and C. Ling, "Smoothing intensity-modulated beam profiles to improve the efficiency of delivery," Med. Phys. 28, 2105-2112 (2001).

${ }^{14}$ E. J. Hall and C. Wu, "Radiation-induced second cancers: The impact of 3D-CRT and IMRT," Int. J. Radiat. Oncol., Biol., Phys. 56, 83-88 (2003).

${ }^{15}$ J. Galvin, G. Ezzell, A. Eisbruch, C. Yu, B. Butler, Y. Xiao, I. Rosen, J. Rosenman, M. Sharpe, L. Xing, P. Xia, T. Lomax, D. Low, and J. Palta, "Implementing IMRT in clinical practice: A joint document of the American Society for Therapeutic Radiology and Oncology and the American Association of Physicists in Medicine," Int. J. Radiat. Oncol., Biol., Phys. 58, 1616-1634 (2004).

${ }^{16} \mathrm{M}$. Alber, and F. Nüsslin, "Optimization of intensity modulated radiotherapy under constraints for static and dynamic MLC delivery," Phys Med. Biol. 46, 3229-3239 (2001).

${ }^{17}$ P. S. Cho, and R. J Marks II, "Hardware-sensitive optimization for intensity modulated radiotherapy," Phys. Med. Biol. 45, 429-440 (2000).

${ }^{18}$ J. Seco, P. M. Evans, and S. Webb, "An optimization algorithm that incorporates IMRT delivery constraints," Phys. Med. Biol. 47, 899-915 (2002).

${ }^{19}$ A. Beavis, P. Ganney, V. Whitton, and L. Xing, "Optimization of the step-and-shoot leaf sequence for delivery of intensity modulated radiation therapy using a variable division scheme," Phys. Med. Biol. 46, 24572465 (2001).

${ }^{20}$ S. M. Crooks, L. F. McAven, D. F. Robinson, and L. Xing, "Minimizing delivery time and monitor units in static IMRT by leaf-sequencing," Phys. Med. Biol. 47, 3105-3116 (2002).

${ }^{21}$ M. Langer, V. Thai, and L. Papiez, "Improved leaf sequencing reduces segments or monitor units needed to delivery IMRT using multileaf collimators," Med. Phys. 28, 2450-2458 (2001).

${ }^{22}$ D. W. Litzenberg, J. M. Moran, and B. A. Fraass, "Incorporation of realistic delivery limitations into dynamic MLC treatment delivery," Med Phys. 29, 810-820 (2002).

${ }^{23}$ Y. Yang and L. Xing, "Incorporating leaf transmission and head scatter corrections into step-and-shoot leaf sequences for IMRT," Int. J. Radiat. Oncol., Biol., Phys. 55, 1121-1134 (2002).

${ }^{24} \mathrm{~L}$. Ma, "Smoothing intensity-modulated treatment delivery under hardware constraints," Med. Phys. 29, 2937-2295 (2002).

${ }^{25} \mathrm{X}$. Sun and P. Xia, "A new smoothing procedure to reduce delivery seg- ments for static MLC-based IMRT planning," Med. Phys. 31, 1158-1165 (2004).

${ }^{26} \mathrm{~S}$. Webb, D. Convery, and P. Evans, "Inverse planning with constraints to generate smoothed intensity-modulated beams," Med. Phys. 43, 27852794 (1998).

${ }^{27}$ B. A. Fraass and D. L. McShan, "3-D treatment planning: I. Overview of a clinical planning system" in The Use of Computers in Radiation Therapy. edited by I. A. D. Bruinvis, F. H. van der Giessen, H. J. van Kleffens, and F. W. Wittkamper (North-Holland, Elsevier Science, Amsterdam, 1987), pp. 273-276.

${ }^{28}$ D. L. McShan and B. A. Fraass, "3-D treatment planning: II. Integration of gray scale images and solid surface graphics," in Ref. 27, pp. 41-44.

${ }^{29}$ B. A. Fraass, D. L. McShan, and K. J. Weeks, "3-D treatment planning: III. Complete beam's-eye-view planning capabilities," in Ref. 27, pp. 193-196.

${ }^{30}$ B. A. Fraass, D. L. McShan, R. K. Ten Haken, and K. M. Hutchins, "3-D treatment planning: V. A Fast 3-D photon calculation model," in Ref. 27, pp. 521-527.

${ }^{31}$ D. L. McShan, B. A. Fraass, and A. S. Lichter, "Full integration of the beam's eye view concept into computerized treatment planning," Int. J. Radiat. Oncol., Biol., Phys. 18, 1485-1494 (1990).

${ }^{32}$ M. L. Kessler, D. L. McShan, M. Epelman, K. A. Vineberg, A. Eisbruch, T. S. Lawrence, and B. A. Fraass, "Costlets: A generalized approach to cost functions for automated optimization," Optimization and Engineering (to be published).

${ }^{33}$ J. H. Kim, N. Dogan, D. L. McShan, and M. L. Kessler, "An AVS-based system for optimization of conformal radiotherapy treatment plans," Proceedings of the 1995 International Advanced Visual Systems User and Developer Conference, Boston MA, 1995, pp. 417-423.

${ }^{34}$ T. R. Mackie, J. W. Scrimger, and J. J. Battista, "A convolution method of calculating dose for 15-MV x rays," Med. Phys. 12, 188-196 (1985).

${ }^{35}$ T. Bortfeld, D. L. Kahler, T. J. Waldron, and A L Boyer, "X-ray field compensation with multileaf collimators," Int. J. Radiat. Oncol., Biol., Phys. 28, 723-730 (1994).

${ }^{36}$ M. L. P. Dirkx, B. J. M. Heijmen, and J. P. C van Santvoort, "Leaf trajectory calculation for dynamic multileaf collimation to realize optimized fluence profiles," Phys. Med. Biol. 43, 1171-1184 (1998).

${ }^{37}$ S. Spirou and C. S. Chui, "Generation of arbitrary intensity profiles by dynamic jaws or multileaf collimators," Med. Phys. 21, 1031-1042 (1994).

${ }^{38}$ J. Stein, T. Bortfeld, B. Dorschel, and W. Schlegel, "Dynamic x-ray compensation for conformal radiotherapy by means of multileaf collimation," Radiother. Oncol. 32, 163-173 (1994).

${ }^{39}$ R. Svensson, P. Kallman, and A. Brahme, "Analytical solution for the dynamic control of multileaf collimators," Phys. Med. Biol. 39, 37-61 (1994).

${ }^{40}$ J. P. C. van Santvoort and B. J. M. Heijmen, "Dynamic leaf collimation without tongue and groove effects," Phys. Med. Biol. 41, 2091-2105 (1996).

${ }^{41}$ A. Pirzkall, M. Carol, B. Pickett, P. Xia, M. Roach III, and L. Verhey, "The effect of beam energy and number of fields on photon-based IMRT for deep-seated targets," Int. J. Radiat. Oncol., Biol., Phys. 53, 434-442 (2002).

${ }^{42}$ J. M. Michalski, J. A. Purdy, K. Winter, M. Roach III, S. Vijayakumar, H. M. Sandler, A. M. Markoe, M. A. Ritter, K. J. Russell, S. Sailer, W. B. Harms, C. A. Perez, R. B. Wilder, G. E. Hanks, and J. D. Cox, "Preliminary report of toxicity following 3D radiation therapy for prostate cancer on 3DOG/RTOG 9406," Int. J. Radiat. Oncol., Biol., Phys. 46, 391-402 (2000).

${ }^{43}$ D. Litzenberg, L. A. Dawson, H. Sandler, M. G. Sanda, D. L. McShan, R. K. Ten Haken, K. L. Lam, K. K. Brock, and J. M. Balter, "Daily prostate targeting using implanted radiopaque markers" Int. J. Radiat. Oncol., Biol., Phys. 52, 699-703 (2002).

${ }^{44}$ T. LoSasso, C. S. Chui, and C. C. Ling, "Physical and dosimetric aspects of a multileaf collimation system used in the dynamic mode for implementing intensity modulated radiotherapy," Med. Phys. 25, 1919-1927 (1998).

${ }^{45}$ X. Sun, P. Xia, and N. Yu, "Effects of the intensity levels and beam map resolutions on static IMRT plans," Med. Phys. 31, 2402-2411 (2004).

${ }^{46}$ E. Nioutsikou, J. Bedford, J. Christian, M. Brada, and S. Webb, "Segmentation of IMRT plans for radical lung radiotherapy delivery with the stepand-shoot technique," Med. Phys. 31, 892-901 (2004). 